\title{
Influence of MWCNTs Doping on the Structure and Properties of PEDOT:PSS Films
}

\author{
Jiao Li, ${ }^{1,2}$ Juncheng Liu,, ${ }^{2}$ Congjie Gao, ${ }^{1}$ Jinling Zhang, ${ }^{2}$ and Hanbin Sun ${ }^{2}$ \\ ${ }^{1}$ College of Chemistry and Chemical Engineering, Ocean University of China, Qingdao 266003, China \\ ${ }^{2}$ School of Materials Science and Engineering, Shandong University of Technology, Zibo 255049, China
}

Correspondence should be addressed to Congjie Gao, gaocjie@mail.hz.zj.cn

Received 26 March 2009; Revised 6 August 2009; Accepted 7 September 2009

Recommended by Mohamed Sabry Abdel-Mottaleb

Poly(3,4-ethylenedioxythiophene):poly(4-styrenesulfonate) (PEDOT:PSS) doped with multi-walled carbon nanotubes (MWCNTs) films is fabricated on quartz substrates by spin coating method. The effects of MWCNTs on the structure and properties of PEDOT:PSS film have been investigated. X-ray diffractometer (XRD) and Fourier transform Raman spectroscopy (FTRM) show that the crystallization behavior and the main chain of PEDOT:PSS are not changed. Atomic force microscopy (AFM) shows that individual nanotubes are well dispersed in the PEDOT:PSS matrix. Moreover, some nanotubes overlap into a net-like structure, forming new conductive channels, which can enhance efficiently the film conductivity. The conductivity of PEDOT:PSS film doped with a lower percentage of MWCNTs $(0.2 \mathrm{wt} \%)$ is $9.16 \mathrm{~S} / \mathrm{cm}$, higher than that of pure PEDOT:PSS film $(0.28 \mathrm{~S} / \mathrm{cm})$, although the optical transmission of PEDOT:PSS decreases a little after the addition of MWCNTs. The interaction between MWCNTs and PEDOT:PSS during melt mixing is also given a possible explanation.

Copyright $\odot 2009$ Jiao Li et al. This is an open access article distributed under the Creative Commons Attribution License, which permits unrestricted use, distribution, and reproduction in any medium, provided the original work is properly cited.

\section{Introduction}

In the recent years, films fabricated from commercially available poly(3,4-ethylenedioxythiophene):poly(4-styrenesulfonate) (PEDOT:PSS) aqueous dispersions have attracted a lot of attention mainly due to their exceptional advantages of high transparency in the visible range, excellent thermal stability, and aqueous solution processibility $[1,2]$. With all these properties, PEDOT:PSS is now widely used in optoelectronic devices. For example, it can be used as a hole transporter layer in organic light-emitting diodes (OLED) [3-5], or as a buffer layer between the ITO and active layers in organic solar cells (OSC) [6-8]. However, the film also suffers low conductivity with about $0.05 \sim 0.8 \mathrm{~S} / \mathrm{cm}[9,10]$, which directly affects the performance of the device $[11,12]$. Therefore, the improvement of the film conductivity has been considered to be an urgent work.

Recently, several strategies have been applied to increase the conductivity of PEDOT:PSS [10, 13-15]. Kim et al. [10] reported that the enhancement of the PEDOT:PSS conductivity could be achieved by adding organic solvent dimethyl sulfoxide or N,N-dimethylformamide. Ouyang et al. $[13,14]$ reported that the addition of ethylene glycol to the aqueous PEDOT:PSS dispersion could induce the change of conductivity, and they proposed that the conductivity enhancement results form the conversion of the PEDOT molecular conformation from "ben structure" to "quioid structure".

Nanotubes can serve as a good filler for conducting polymer. It has been reported that incorporating of nanotubes into conventional conducting polymers improves the conductivity of nanotubes/polymer composites by many orders of magnitude [15-20], which could be attributed to nanotubes' large contact area, high dimensional aspect ratio, and exceptional electrical conductivity [21].

In this work, the PEDOT:PSS doped with multiwalled carbon nanotubes (MWCNTs) transparent conducting film was fabricated on quartz substrates by spin coating method. The pristine and doped PEDOT:PSS films were characterized by XRD, FTRM, AFM, UV-Vis spectroscopy, and four-point measurement. The effects of MWCNTs on the structure and properties of the PEDOT:PSS film were also investigated. 


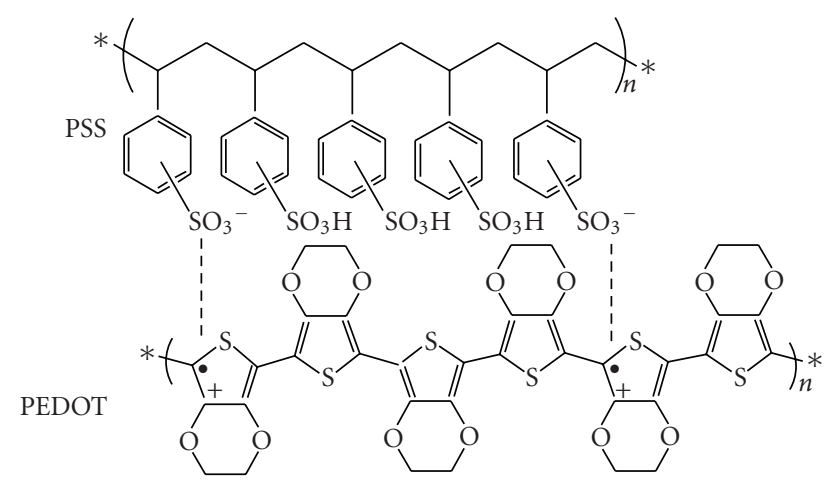

FIGURE 1: Chemical structure of PEDOT:PSS. The "dot" and "plus" represent the unpaired electron and positive charge on the PEDOT chain, respectively.

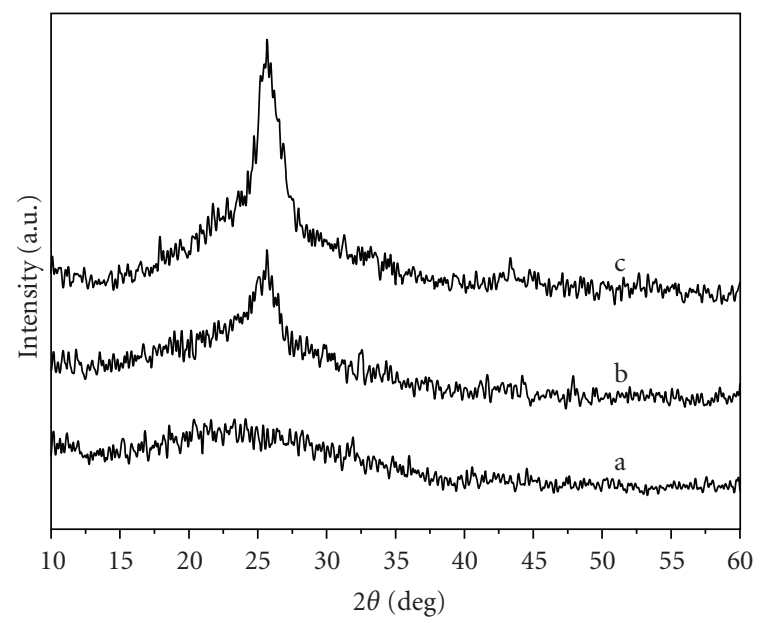

FIGURE 2: XRD patterns of (a) pure PEDOT:PSS, (b) MPEDOT:PSS (0.1 wt \%), and (c) MWCNTs films.

\section{Experimental}

Multiwalled carbon nanotubes (diameter: $10 \sim 30 \mathrm{~nm}$, length: $5 \sim 15 \mu \mathrm{m}$, purity: $>95 \%$ ) were purchased from Beijing Nachen Nanotech Co., Ltd and were further acidtreated by the previously established methods $[22,23]$. The MWCNTs after acid-treatment can be dispersed into aqueous PEDOT:PSS solution and no precipitation is observed in the solution after several weeks. PEDOT:PSS aqueous solution ( $1.3 \mathrm{wt} \%$ dispersed in $\mathrm{H}_{2} \mathrm{O}$ ) was from Aldrich. Other reagents and solvents were of analytical grade.

The chemical structure of PEDOT:PSS is shown in Figure 1. In the PEDOT:PSS dispersion, PEDOT is the charge transporting species $[9,10]$. PSS acts as a charge-compensating counterion to stabilize the $\mathrm{p}$-doped conducting polymer, and forms a processable water-borne dispersion of negatively charged swollen colloidal particles consisting of PEDOT and excess PSS [24, 25].

All the quartz substrates $(20 \mathrm{~mm} \times 20 \mathrm{~mm})$ were ultrasonically cleaned with a series of organic solvents (ethanol, methanol, and acetone), then rinsed in ultrasonic bath with deionized water, and dried in a vacuum oven. Residual organic contaminations were subsequently removed by exposing to a UV-ozone lamp for $30 \mathrm{~min}$. Prior to spin coating, the precursor solution of PEDOT:PSS with appropriate mass of MWCNTs was prepared. Some amount of acid treated-MWCNTs were added to the PEDOT:PSS aqueous dispersion in ultrasonic bath for about 12 hours. The PEDOT:PSS and acid treated-MWCNTs-doped PEDOT:PSS films (PEDOT:PSS and M-PEDOT:PSS, separately) with a thickness of $95-100 \mathrm{~nm}$, as determined with scanning electron microscopy, were fabricated on transparent quartz substrates by spin coating method. The spin coating procedure included 20 seconds of $2000 \mathrm{rpm}$ followed by 30 seconds of $5000 \mathrm{rmp}$. Then these films were dried at $110^{\circ}$ for 60 minutes in the vacuum oven before any further characterization.

The molecular structure analysis of all the samples was carried out with Fourier transform Raman spectroscopy, which was recorded with a RFS 100 Bruker spectrometer (excitation wavelength $1064 \mathrm{~nm}$ ). Sample structures were analyzed with D8 Advance Brucker AXS X-ray diffractometer, with $\mathrm{Cu} \mathrm{K}_{\alpha 1}$ radiation. The dispersion of acid-treated MWCNTs in PEDOT:PSS matrix was investigated with atomic force microscopy, which was performed with Digital Instruments Nanoscope III operating in tapping mode. The optical properties were characterized with a TU-1901 Dualbeam UV-Visible spectrophotometer. The conductivity of the films was measured by the four-point probe technique with a Keithley 2400 Source Meter. All the measurements were performed at room temperature.

\section{Results and Discussion}

The XRD patterns of pure MWCNTs, PEDOT:PSS, and M-PEDOT:PSS films are shown in Figure 2. There are not any sharp peaks in the pristine PEDOT:PSS profile (Figure 2(a)), which indicates substantial amorphous nature of the PEDOT:PSS film. Similar results were also obtained by different research groups $[26,27]$. The pure MWCNTs film shows a sharp peak centered on $2 \theta$ value of $25.6^{\circ}$ corresponding to the $\left(\begin{array}{lll}0 & 0 & 2\end{array}\right)$ planes of MWCNTs (Figure $\left.2(\mathrm{c})\right)$. The composite film (Figure 2(b)) shows the characteristic peaks of both MWCNTs and PEDOT:PSS without any additional 


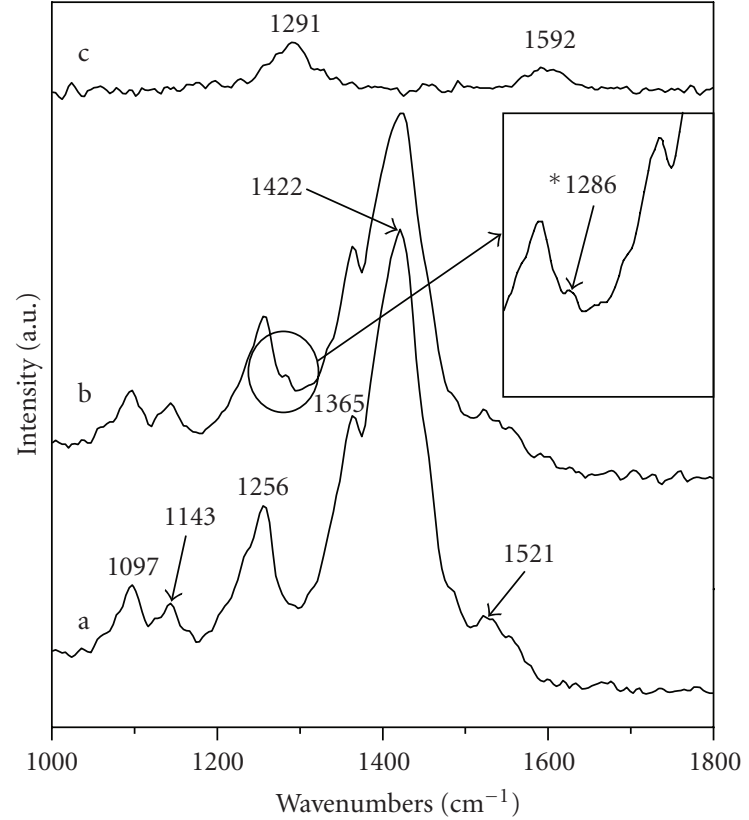

FIGURE 3: Raman spectra of (a) pure PEDOT:PSS, (b) MPEDOT:PSS (0.1 wt \%), and (c) MWCNTs films. * The inset curve shows clearly the peak of $1286 \mathrm{~cm}^{-1}$.

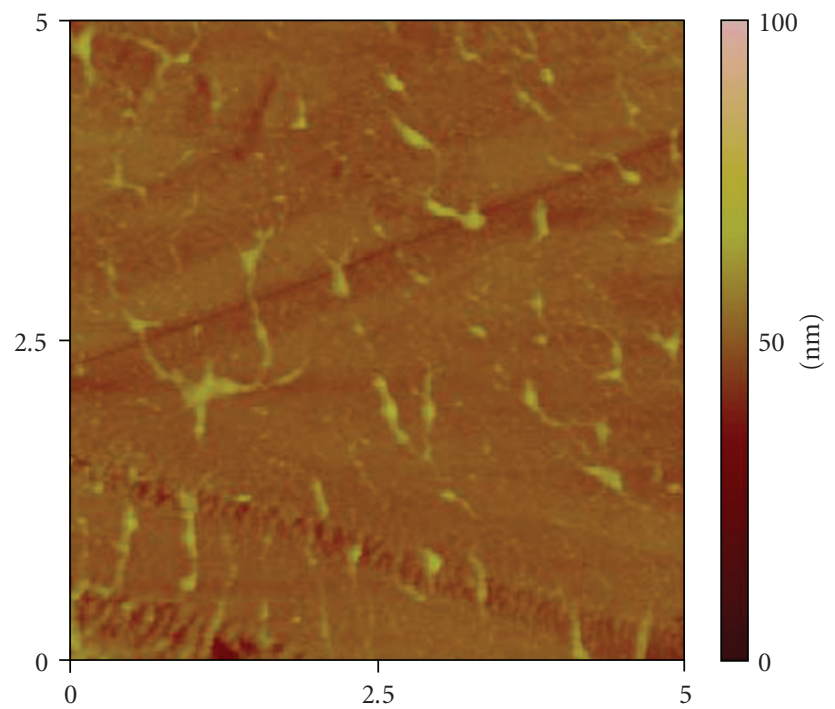

FIGURE 4: AFM image $(5 \times 5 \mu \mathrm{m})$ of M-PEDOT:PSS $(0.1 \mathrm{wt} \%)$ film.

bands, which represents the absence of covalent interactions between the phases. Moreover, the crystallization behavior of the PEDOT:PSS film is not changed.

The Raman spectrum of pure PEDOT:PSS film is identified in [28-30] (Figure 3(a)). Raman spectrum shows six dominant principal peaks of PEDOT. The peak at $1521 \mathrm{~cm}^{-1}$ represents $\mathrm{C}_{\alpha}=\mathrm{C}_{\beta}$ asymmetric vibrations; $1421 \mathrm{~cm}^{-1}$ can be assigned to $\mathrm{C}_{\alpha}=\mathrm{C}_{\beta}$ symmetric vibrations, $1365 \mathrm{~cm}^{-1}$ to $\mathrm{C}-\mathrm{C}$ stretching deformations, $1256 \mathrm{~cm}^{-1}$ to $\mathrm{C}-\mathrm{C}$ in-plane symmetric stretching, and $1143 \mathrm{~cm}^{-1}$ and $1097 \mathrm{~cm}^{-1}$ to C$\mathrm{C}$ in-plane bending. As shown in Figure 3(c), there are mainly two optically active phonon modes in the firstorder Raman spectrum of MWCNTs film, and two peaks at $1592 \mathrm{~cm}^{-1}$ and $1291 \mathrm{~cm}^{-1}$ represent a graphite $\mathrm{E}_{2 \mathrm{~g}}$ mode and an MWCNTs disordered $A_{1 g}$ mode, respectively. The disordered $\mathrm{A}_{\mathrm{lg}}$ mode indicates the imperfection of the $s p^{2}$ hybridization of MWCNTs, which may occur during the production process or the acid-treated process [31]. Raman spectrum of the M-PEDOT:PSS film is essentially the same as that of PEDOT:PSS film except one additional peak centered at $1286 \mathrm{~cm}^{-1}$ (Figure 3(b)).

Chemical and physical interactions between some materials and MWCNTs often occur during melt mixing, and these interactions can affect the Raman spectrum of MWCNTs. These phenomena have been confirmed by both theoretical and experimental researches $[32,33]$. Combining with the results of XRD, we speculate that there may be some noncovalent interactions, which might stem from the $\pi-\pi$ interaction between the delocalized $\pi$ bond network of MWCNTs and the thiophene rings of PEDOT backbone. This conjugated interaction would affect the electronic density among MWCNTs. Therefore, the peak of MWCNTs at $1291 \mathrm{~cm}^{-1}$, which shifts red from $1291 \mathrm{~cm}^{-1}$ to $1286 \mathrm{~cm}^{-1}$, may result from the change of electronic density of MWCNTs. While the peak of MWCNTs at $1592 \mathrm{~cm}^{-1}$ is so weak that it cannot be observed (Figure 3(b)). However, the mechanism of the interactions between MWCNTs and PEDOT:PSS during melt mixing is still an assumption and needs to be further studied.

AFM image $(5 \times 5 \mu \mathrm{m})$ in Figure 4 shows that individual nanotubes are well dispersed in the PEDOT:PSS matrix. Some nanotubes overlap into a net-like structure, which implies that some continuous channels are embedded in the PEDOT:PSS matrix.

When PEDOT:PSS is applied in OLED or OSC devices, it is always important to keep its transmittance high. Figure 5 shows the wavelength dependence of the optical transparency of PEDOT:PSS and M-PEDOT:PSS thin films on the wavelength range from $550 \mathrm{~nm}$ to $950 \mathrm{~nm}$, which corresponds to the power density of ambient sunlight on the Earth's surface [34]. The PEDOT:PSS film gives a good optical transmittance in the above wavelength range (93.2\% average) (Figure 5(a)). Moreover, The transmittance increases from $950 \mathrm{~nm}$ to $550 \mathrm{~nm}$, which is mainly because of more absorptions in the infrared region for the PEDOT:PSS film $[5,35]$, as shown in Figure 5(b). Transmittance curve of M-PEDOT:PSS film is about $88.5 \%$ average, about $5 \%$ less than that of pure PEDOT:PSS film. This may result from the formation of the conductive network of MWCNTs and some absorptions of MWCNTs in the above wavelength range $[24,36]$.

As shown in Figure 6, the conductivity of pure PEDOT:PSS film is $0.28 \mathrm{~S} / \mathrm{cm}$. While the conductivity increases drastically when MWCNTs doping concentration increases from $0.04 \mathrm{wt} \%$ to $0.12 \mathrm{wt} \%$, and then increases moderately at loading levels in excess of $0.12 \mathrm{wt} \%$. When MWCNTs content reaches about $0.2 \mathrm{wt} \%$, the conductivity of composite film is $9.16 \mathrm{~S} / \mathrm{cm}$. These indicate that the percolation threshold of the nanocompostie films resides between $0.04 \mathrm{wt} \%$ and $0.12 \mathrm{wt} \%$, where some conductive 


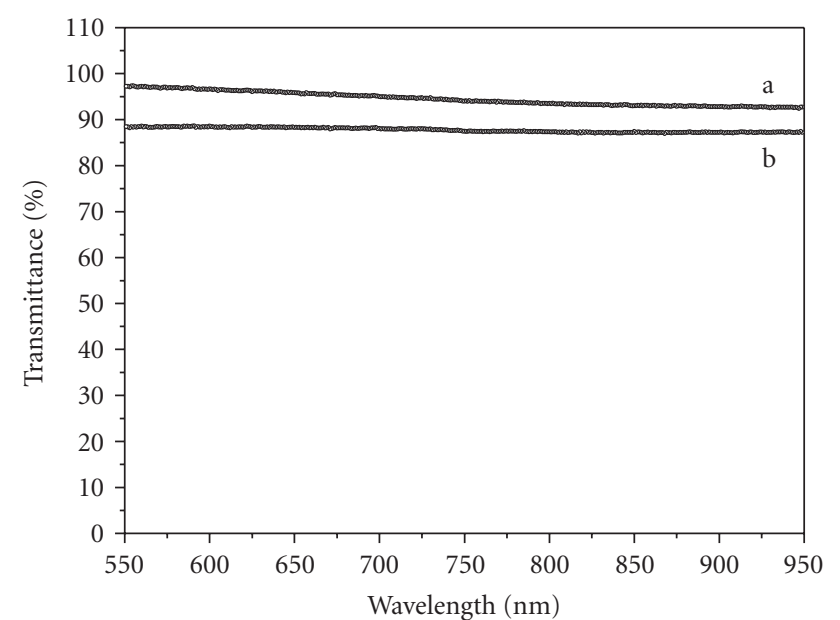

Figure 5: Transmittance of (a) pure PEDOT:PSS and (b) MPEDOT:PSS (0.1 wt\%) films.

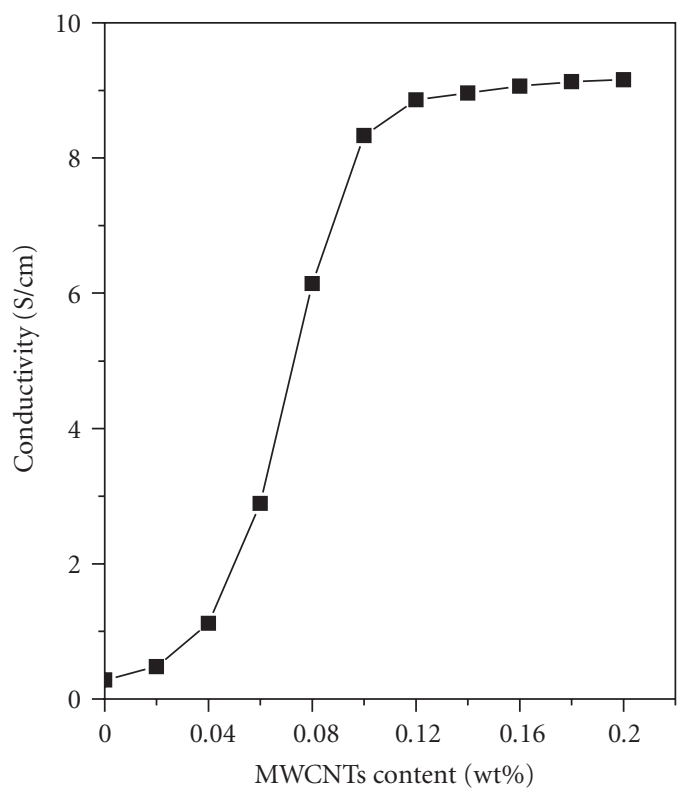

Figure 6: The electrical conductivity of composite films as a function of weight fraction of MWCNTs.

MWCNT channels in the PEDOT:PSS matrix might form, as shown in AFM (Figure 4), which can help charge transport and enhance the conductivity of films $[37,38]$.

\section{Conclusion}

In summary, influences of MWCNTs doping on the structure and properties of PEDOT:PSS films have been investigated. XRD and FTRM show that the crystallization behavior and the main chain of PEDOT:PSS are not changed. AFM shows that individual nanotubes are well dispersed in the PEDOT:PSS matrix. Moreover, some nanotubes overlap into a net-like structure, forming new conductive channels, which can enhance efficiently the film conductivity.
The conductivity of PEDOT:PSS film doped with a lower percentage of MWCNTs $(0.2 \mathrm{wt} \%)$ is $9.16 \mathrm{~S} / \mathrm{cm}$, higher than that of pure PEDOT:PSS film $(0.28 \mathrm{~S} / \mathrm{cm})$, although the optical transmission of PEDOT:PSS decreases a little after the addition of MWCNTs. The interaction between MWCNTs and PEDOT:PSS during melt mixing is also given a possible explanation. However, this explanation is still an assumption and needs to be further studied.

\section{Acknowledgment}

Financial support from the program for New Century Excellent Talents in University (NCET, Grant no. NCET-040648 ) is gratefully acknowledged.

\section{References}

[1] G. Heywang and F. Jonas, "Poly(alkylenedioxythiophene)snew, very stable conducting polymers," Advanced Materials, vol. 4, no. 2, pp. 116-118, 1992.

[2] L. Groenendaal, F. Jonas, D. Freitag, H. Pielartzik, and J. R. Reynolds, "Poly(3,4-ethylenedioxythiophene) and its derivatives: past, present, and future," Advanced Materials, vol. 12, no. 7, pp. 481-494, 2000.

[3] Y.-F. Zhou, Y.-B. Yuan, L.-F. Cao, et al., "Improved stability of OLEDs with mild oxygen plasma treated PEDOT:PSS," Journal of Luminescence, vol. 122-123, pp. 602-604, 2007.

[4] T.-C. Tsai, W.-Y. Hung, L.-C. Chi, K.-T. Wong, C.-C. Hsieh, and P.-T. Chou, "A new ambipolar blue emitter for NTSC standard blue organic light-emitting device," Organic Electronics, vol. 10, no. 1, pp. 158-162, 2009.

[5] P. Vacca, M. Petrosino, R. Miscioscia, et al., "Poly(3,4ethylenedioxythiophene):poly(4-styrenesulfonate)ratio: structural, physical and hole injection properties in organic light emitting diodes," Thin Solid Films, vol. 516, no. 12, pp. 42324237, 2008.

[6] C.-J. Ko, Y.-K. Lin, F.-C. Chen, and C.-W. Chu, "Modified buffer layers for polymer photovoltaic devices," Applied Physics Letters, vol. 90, no. 6, Article ID 063509, 3 pages, 2007.

[7] J. Bouclé, S. Chyla, M. S. P. Shaffer, J. R. Durrant, D. D. C. Bradley, and J. Nelson, "Hybrid solar cells from a blend of poly(3-hexylthiophene) and ligand-capped $\mathrm{TiO}_{2}$ nanorods," Advanced Functional Materials, vol. 18, no. 4, pp. 622-633, 2008.

[8] K. X. Steirer, M. O. Reese, B. L. Rupert, et al., "Ultrasonic spray deposition for production of organic solar cells," Solar Energy Materials \& Solar Cells, vol. 93, no. 4, pp. 447-453, 2009.

[9] S. K. M. Jönsson, J. Birgerson, X. Crispin, et al., “The effects of solvents on the morphology and sheet resistance in poly $(3,4-$ ethylenedioxythiophene)-polystyrenesulfonic acid (PEDOTPSS) films," Synthetic Metals, vol. 139, no. 1, pp. 1-10, 2003.

[10] J. Y. Kim, J. H. Jung, D. E. Lee, and J. Joo, "Enhancement of electrical conductivity of poly(3,4-ethylenedioxythiophene)/poly(4-styrenesulfonate) by a change of solvents," Synthetic Metals, vol. 126, no. 2-3, pp. 311-316, 2002.

[11] C. C. Oey, A. B. Djurišić, C. Y. Kwong, et al., "Nanocomposite hole injection layer for organic device applications," Thin Solid Films, vol. 492, no. 1-2, pp. 253-258, 2005.

[12] J. Huang, P. F. Miller, J. S. Wilson, A. J. de Mello, J. C. de Mello, and D. D. C. Bradley, "Investigation of the effects of doping and post-deposition treatments on the conductivity, 
morphology, and work function of poly(3,4-ethylenedioxythiophene)/poly(styrene sulfonate) films," Advanced Functional Materials, vol. 15, no. 2, pp. 290-296, 2005.

[13] J. Ouyang, Q. Xu, C.-W. Chu, Y. Yang, G. Li, and J. Shinar, "On the mechanism of conductivity enhancement in poly $(3,4-$ ethylenedioxythiophene):poly(styrene sulfonate) film through solvent treatment," Polymer, vol. 45, no. 25, pp. 8443-8450, 2004.

[14] J. Ouyang, C.-W. Chu, F.-C. Chen, Q. Xu, and Y. Yang, "Polymer optoelectronic devices with high-conductivity poly $(3,4-$ ethylenedioxythiophene) anodes," Journal of Macromolecular Science Part A, vol. 41, no. 12, pp. 1497-1511, 2004.

[15] H. Peng and X. Sun, "Highly aligned carbon nanotube/polymer composites with much improved electrical conductivities," Chemical Physics Letters, vol. 471, no. 1-3, pp. 103-105, 2009.

[16] Z. Yang, H. Pu, J. Yuan, D. Wan, and Y. Liu, "PhthalocyaninesMWCNT hybrid materials: fabrication, aggregation and photoconductivity properties improvement," Chemical Physics Letters, vol. 465, no. 1-3, pp. 73-77, 2008.

[17] M.-C. Wu, Y.-Y. Lin, S. Chen, et al., "Enhancing light absorption and carrier transport of P3HT by doping multiwall carbon nanotubes," Chemical Physics Letters, vol. 468, no. 1-3, pp. 64-68, 2009.

[18] K. R. Reddy, B. C. Sin, K. S. Ryu, J.-C. Kim, H. Chung, and Y. Lee, "Conducting polymer functionalized multi-walled carbon nanotubes with noble metal nanoparticles: synthesis, morphological characteristics and electrical properties," Synthetic Metals, vol. 159, no. 7-8, pp. 595-603, 2009.

[19] T.-M. Wu, H.-L. Chang, and Y.-W. Lin, "Synthesis and characterization of conductive polypyrrole/multi-walled carbon nanotubes composites with improved solubility and conductivity," Composites Science and Technology, vol. 69, no. 5, pp. 639-644, 2009.

[20] Y. Ma, S. R. Ali, A. S. Dodoo, and H. He, "Enhanced sensitivity for biosensors: multiple functions of DNA-wrapped single-walled carbon nanotubes in self-doped polyaniline nanocomposites," Journal of Physical Chemistry B, vol. 110, no. 33, pp. 16359-16365, 2006.

[21] R. H. Baughman, A. A. Zakhidov, and W. A. de Heer, "Carbon nanotubes: the route toward applications," Science, vol. 297, no. 5582, pp. 787-792, 2002.

[22] G.-F. Wang, X.-M. Tao, and R.-X. Wang, "Fabrication and characterization of OLEDs using PEDOT:PSS and MWCNT nanocomposites," Composites Science and Technology, vol. 68, no. 14, pp. 2837-2841, 2008.

[23] R. A. Hatton, N. P. Blanchard, L. W. Tan, G. Latini, F. Cacialli, and S. R. P. Silva, "Oxidised carbon nanotubes as solution processable, high work function hole-extraction layers for organic solar cells," Organic Electronics, vol. 10, no. 3, pp. 388395, 2009.

[24] S. Ghosh and O. Inganäs, "Self-assembly of a conducting polymer nanostructure by physical crosslinking: applications to conducting blends and modified electrodes," Synthetic Metals, vol. 101, no. 1-3, pp. 413-416, 1999.

[25] F. Jonas and G. Heywang, "Technical applications for conductive polymers," Electrochimica Acta, vol. 39, no. 8-9, pp. 13451347, 1994.

[26] L. Zhan, Z. Song, J. Zhang, et al., "PEDOT: cathode active material with high specific capacity in novel electrolyte system," Electrochimica Acta, vol. 53, no. 28, pp. 8319-8323, 2008.
[27] C. Zhou, S. Wang, Q. Zhuang, and Z. Han, "Enhanced conductivity in polybenzoxazoles doped with carboxylated multi-walled carbon nanotubes," Carbon, vol. 46, no. 9, pp. 1232-1240, 2008.

[28] M. Łapkowski and A. Proń, "Electrochemical oxidation of poly(3,4-ethylenedioxythiophene)— “in situ" conductivity and spectroscopic investigations," Synthetic Metals, vol. 110, no. 1, pp. 79-83, 2000.

[29] B.-H. Wang, Y.-H. Deng, J. Ge, X. Zhou, X.-G. Wang, and B.-Z. Yang, "Chemical synthesis of poly (3,4ethylenedioxythiophene) in three different solvents," Journal of Functional Materials, vol. 36, no. 10, pp. 1610-1612, 2005 (Chinese).

[30] S. Garreau, G. Louarn, J. P. Buisson, G. Froyer, and S. Lefrant, "In situ spectroelectrochemical Raman studies of poly $(3,4-$ ethylenedioxythiophene) (PEDT)," Macromolecules, vol. 32, no. 20, pp. 6807-6812, 1999.

[31] W. Jarernboon, S. Pimanpang, S. Maensiri, E. Swatsitang, and V. Amornkitbamrung, "Effects of multiwall carbon nanotubes in reducing microcrack formation on electrophoretically deposited $\mathrm{TiO}_{2}$ film," Journal of Alloys and Compounds, vol. 476, no. 1-2, pp. 840-846, 2009.

[32] G. Q. Tang, H. M. Wang, S. S. Jin, et al., "Interaction between multi-walled carbon nanotubes and bromime and conducting mechanism," Acta Chimica Sinica, vol. 66, no. 6, pp. 675-679, 2008.

[33] H.-X. Wu, R. Tong, X.-Q. Qiu, et al., "Functionalization of multiwalled carbon nanotubes with polystyrene under atom transfer radical polymerization conditions," Carbon, vol. 45, no. 1, pp. 152-159, 2007.

[34] B. R. Saunders and M. L. Turner, "Nanoparticle-polymer photovoltaic cells," Advances in Colloid and Interface Science, vol. 138, no. 1, pp. 1-23, 2008.

[35] L. A. A. Pettersson, S. Ghosh, and O. Inganäs, "Optical anisotropy in thin films of poly(3,4-ethylenedioxythiophene)poly(4-styrenesulfonate)," Organic Electronics, vol. 3, no. 3-4, pp. 143-148, 2002.

[36] J. K. W. Sandler, J. E. Kirk, I. A. Kinloch, M. S. P. Shaffer, and A. H. Windle, "Ultra-low electrical percolation threshold in carbon-nanotube-epoxy composites," Polymer, vol. 44, no. 19, pp. 5893-5899, 2003.

[37] S. Kumar, T. D. Dang, F. E. Arnold, et al., "Synthesis, structure, and properties of $\mathrm{PBO} / \mathrm{SWNT}$ composites," Macromolecules, vol. 35, no. 24, pp. 9039-9043, 2002.

[38] J. Zhang, M. Mine, D. Zhu, and M. Matsuo, "Electrical and dielectric behaviors and their origins in the three-dimensional polyvinyl alcohol/MWCNT composites with low percolation threshold," Carbon, vol. 47, no. 5, pp. 1311-1320, 2009. 


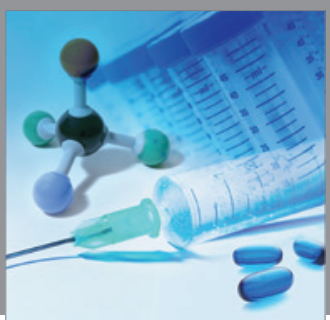

International Journal of

Medicinal Chemistry

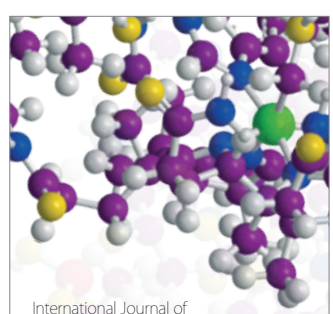

Carbohydrate Chemistry

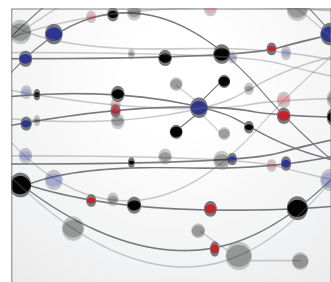

The Scientific World Journal
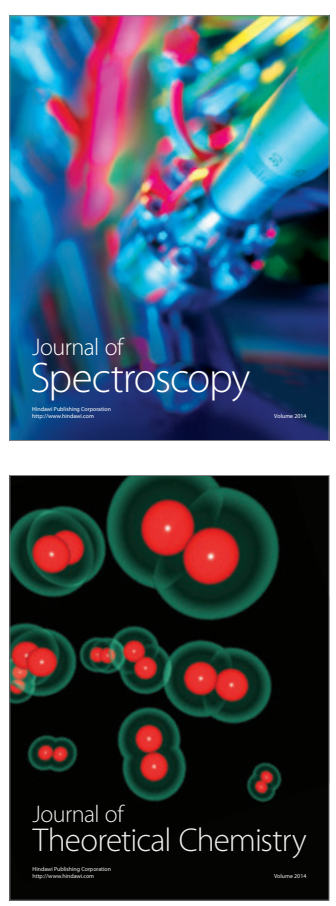
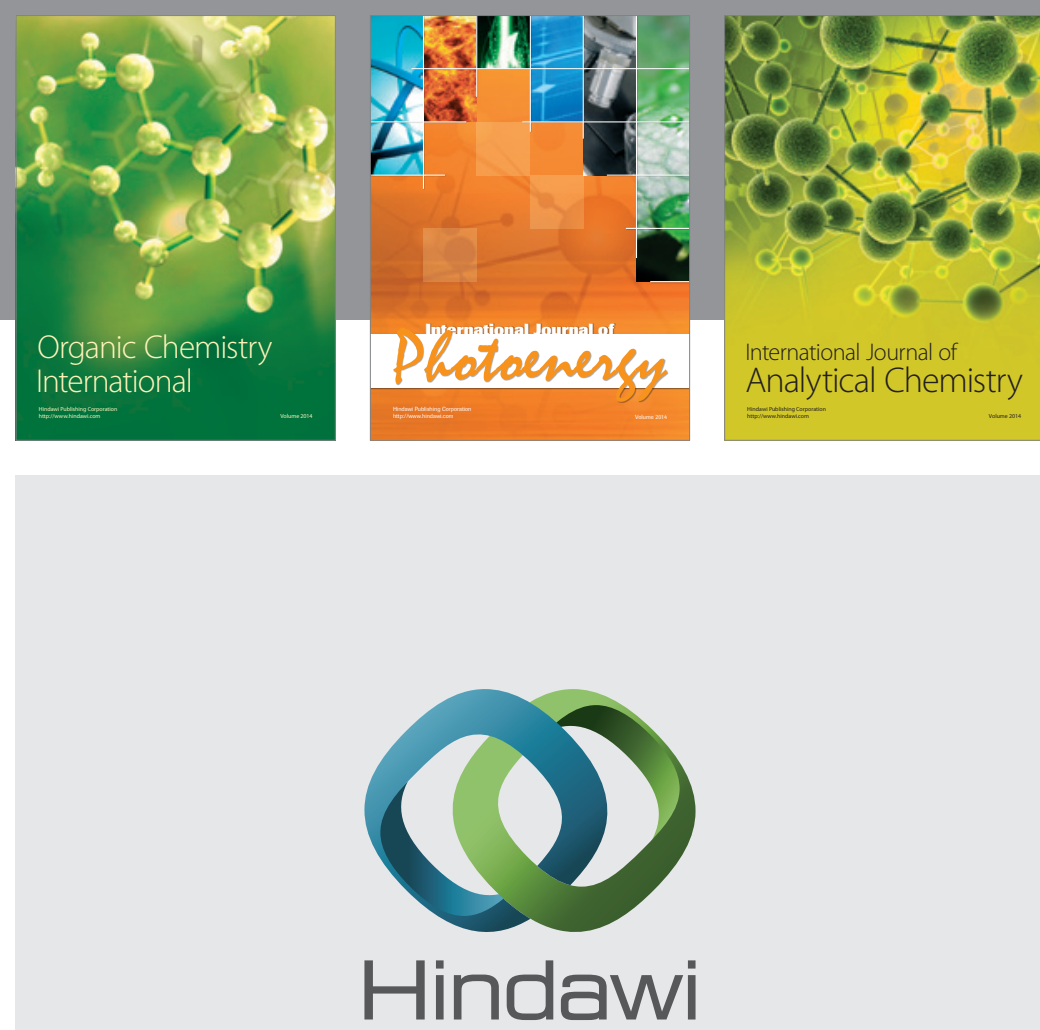

Submit your manuscripts at

http://www.hindawi.com
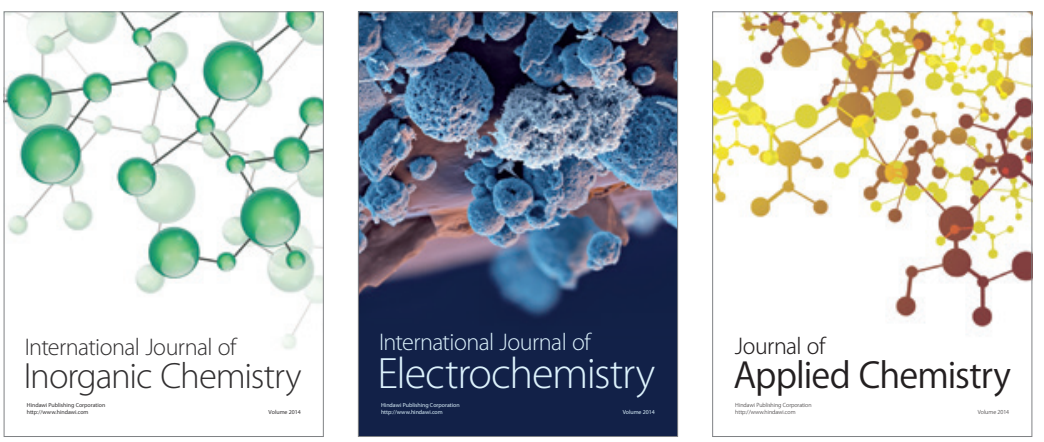

Journal of

Applied Chemistry
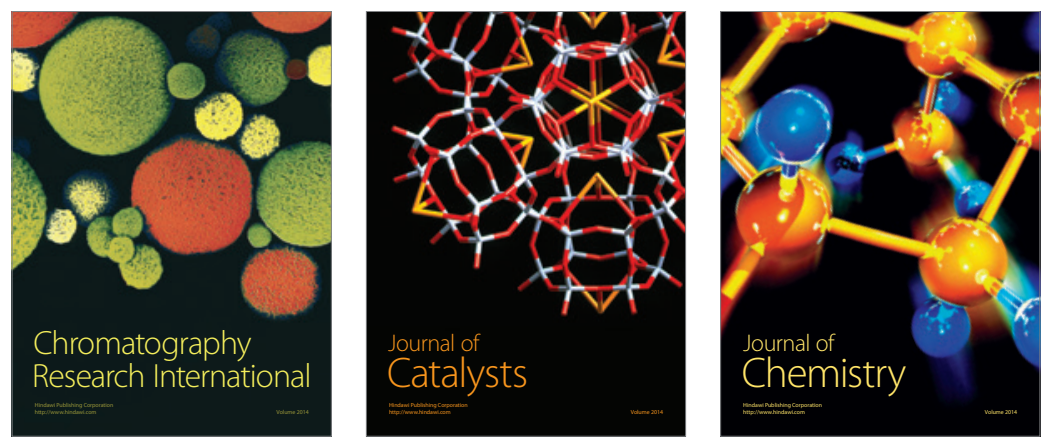
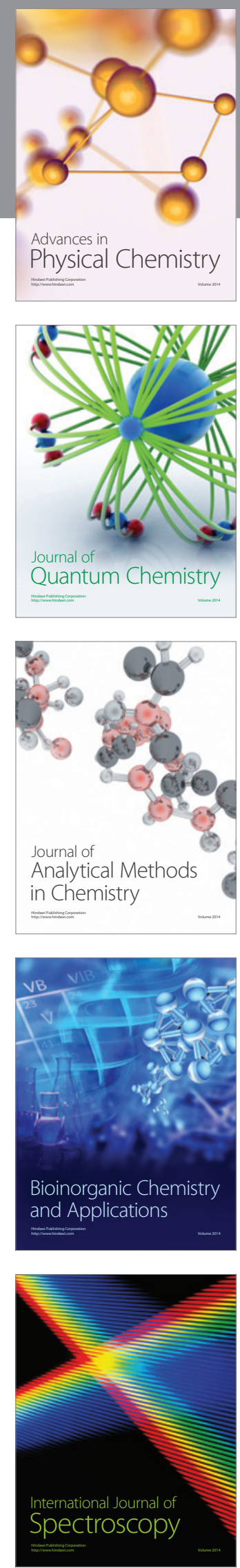